\title{
Converting and Standardizing Various Measures of Arterial Stiffness to Pulse Wave Velocity
}

\author{
Taha Alhalimi Jisok Lim Drew Gourley Hirofumi Tanaka \\ Department of Kinesiology and Health Education, Cardiovascular Aging Research Laboratory, \\ The University of Texas at Austin, Austin, TX, USA
}

\section{Keywords}

Arterial distensibility · Conversion equation - Vascular function · Aging · Meta-analysis

\begin{abstract}
Background: A variety of arterial stiffness measures have been used to assess the impacts of disease states and various interventions without clear consensus among them. One of the primary problems faced by investigators conducting systematic reviews and meta-analyses is the lack of standardized methodology with a same unit to evaluate and compare investigations using different arterial stiffness measures. Therefore, the purpose of this study was to derive and summarize standardized equations to convert commonly used image-based measures of arterial stiffness to local pulse wave velocity (PWV). Methods: We first conducted a literature search to obtain and summarize conversion equations in the published literature such that these equations can be found in one convenient location. Then, we generated regression equations using the data collected in a well-controlled laboratory-based study, in which all measures of arterial stiffness were obtained in 49 apparently healthy participants. Results: All literature-based conver-
\end{abstract}

sion equations produced similar local PWV values and were moderately and significantly correlated with directly measured carotid-femoral PWV (cfPWV) with a Pearson's $r$ ranging from 0.41 to 0.50 . The local PWV using laboratory-based equations were modestly associated with cfPWV $(r=0.39$ 0.49 ) with an exception of incremental elastic modulus ( $r=$ $0.15, p>0.05)$. Conclusion: Commonly used measures of ultrasound-based arterial stiffness can be converted to local PWV and compared with a reference standard measure of arterial stiffness.

(c) 2021 S. Karger AG, Basel

\section{Introduction}

Arterial stiffness reflects the physical properties of the arterial wall that play a crucial role in regulating blood pressure and pulsatile stress after each cardiac contraction [1]. Most of the important risk factors, including hypertension, hyperlipidemia, smoking, sedentary lifestyle, and aging, have all been associated with increased arterial stiffness [2,3]. Indeed, elevated arterial stiffness is an independent risk factor for cardiovascular disease (CVD) and mortality $[2,3]$. 
Arterial stiffness can be measured using a variety of techniques [4-9]. The transmission model such as pulse wave velocity (PWV) evaluates the arterial stiffness using pulse transit time between 2 points. The stiffer arteries prevent the expansion within the arterial wall and the kinetic energy travels longitudinally along the artery at a faster rate than healthy arterial wall $[10,11]$. Carotidfemoral PWV (cfPWV) has been utilized as the reference standard measure of arterial stiffness due to its well-established link to CVD and mortality [7]. Another common method of measuring arterial stiffness is pulsation model, which uses imaging techniques, such as ultrasound or magnetic resonance imaging [8]. These imagebased techniques when they combined with local blood pressure are direct measures of the physical properties of the arterial wall but can yield a variety of arterial stiffness indices including arterial distensibility (AD), arterial compliance (AC), and $\beta$-stiffness index $[1,9,12]$.

Currently, the literature is filled with different arterial stiffness measures with each technique being expressed with different units and characterized different vascular beds. These unique features of diverse arterial stiffness measurements have provided difficulties in comparing investigations using different techniques and synthesizing and conducting meta-analyses and systematic reviews of arterial stiffness. Previous meta-analyses had to restrict the inclusion to only studies reporting PWV $[13,15]$ or rely on the random effect models to statistically account for the heterogeneity of measures [16, 18]. Accordingly, the primary aim of the present study was to identify, create, and then evaluate standardized conversion equations to transform common image-based measures of arterial stiffness (AC, distensibility, $\beta$-stiffness index, and elastic modulus) to local PWV. We reasoned that these conversion equations could be utilized by epidemiologists and meta-analysts to compare and synthesize studies using various measures of arterial stiffness.

\section{Material and Methods}

In order to convert ultrasound-derived measures of arterial stiffness to local PWV, we used 2 different but complementary approaches: the literature-based searches and laboratory-based regression equations.

\section{Participants}

For the laboratory-based study, 49 participants varying in age (21-78 years) were studied. All the participants were apparently healthy without any known CVD or diabetes. None of them were chronic smoker, pregnant, or taking any cardiovascular medications (Table 1). Participants were instructed to abstain from stren- uous exercise for $12 \mathrm{~h}$, caffeine consumption for $6 \mathrm{~h}$, and food for $4 \mathrm{~h}$ prior to experimental trials. The Institutional Review Board at University of Texas at Austin approved this study. Written informed consent was obtained from all participants.

\section{Measurements}

Arterial blood pressure was measured using the oscillometric device (Omron Healthcare, Kyoto, Japan) on the brachial artery. cfPWV was measured using the vascular testing device (VP1000plus, Omron Healthcare, Kyoto, Japan) equipped with oscillometric pressure sensor cuffs, electrocardiograms, phonocardiograms, and arterial applanation tonometry sensors [18]. The simultaneous measurement of ultrasound imaging of the common carotid artery and applanation tonometrically obtained arterial pressure waveforms from the contralateral carotid artery and calibrated using brachial mean and diastolic pressure to allow noninvasive determination of local arterial stiffness [19]. Common carotid artery diameter was measured from the images derived from an ultrasound machine equipped with a high-resolution linear array transducer (Philips iE33 Ultrasound System, Bothel, WA, USA). Carotid artery diameter was analyzed using image analysis software (Brachial Analyzer, Medical Imaging Applications, Coralville, IA, USA), and pressure waveforms of the carotid artery were analyzed using a waveform analysis software (WinDq 2000, Dataq Instruments, Akron, OH, USA). The following image-based measures of arterial stiffness were evaluated in the present study.

AC

$$
\mathrm{AC}=\frac{\Delta D}{\Delta P}
$$

Cross-sectional compliance

$$
\mathrm{CC}=\frac{\pi\left(2 D_{\mathrm{d}} \times \Delta D+\Delta D^{2}\right)}{4 \Delta P}
$$

$\mathrm{AD}$

$$
\mathrm{AD}=\frac{\Delta D}{\Delta P \times D_{\mathrm{d}}}
$$

Distensibility coefficient (DC)

$$
\mathrm{DC}=\frac{\left(2 D_{\mathrm{d}} \times \Delta D+\Delta D^{2}\right)}{\Delta P \times D_{\mathrm{d}}^{2}}
$$

Peterson's Modulus $\left(E_{\mathrm{p}}\right)$

$$
E_{\mathrm{p}}=\frac{\Delta P \times D_{\mathrm{d}}}{\Delta D}
$$

Incremental elastic Modulus $\left(E_{\text {inc }}\right)$

$$
E_{\text {inc }}=\frac{\Delta P \times D_{\mathrm{d}}^{2}}{\Delta D \times 2 \mathrm{IMT}}
$$

$\beta$-stiffness index $(\beta$-SI)

$$
\beta-\mathrm{SI}=\ln \left(\frac{\text { Systolic BP }}{\text { Diastolic BP }}\right) \times \frac{D_{\mathrm{d}}}{\Delta D}
$$


Table 1. Selected participant characteristics

\begin{tabular}{|c|c|}
\hline Variable & Means \pm SD or $n$ \\
\hline Age, year & $46 \pm 19$ \\
\hline Sex, $n$ & $27 \mathrm{M} / 22 \mathrm{~F}$ \\
\hline Height, $m$ & $1.71 \pm 0.09$ \\
\hline Body weight, kg & $76 \pm 16$ \\
\hline $\mathrm{BMI}, \mathrm{kg} / \mathrm{m}^{2}$ & $26 \pm 4$ \\
\hline Carotid systolic BP, mm Hg & $105 \pm 11$ \\
\hline Carotid diastolic BP, mm Hg & $75 \pm 8$ \\
\hline Carotid PP, $\mathrm{mm} \mathrm{Hg}$ & $30 \pm 8$ \\
\hline Carotid artery diameter, $\mathrm{m} \times 10^{-3}$ & $7.1 \pm 0.6$ \\
\hline $\mathrm{IMT}, \mathrm{m} \times 10^{-3}$ & $0.51 \pm 0.13$ \\
\hline $\mathrm{CC} \mathrm{m}{ }^{2} / \mathrm{Pa} \times 10^{-10}$ & $17.6 \pm 0.7$ \\
\hline $\mathrm{AC}, \mathrm{m} / \mathrm{Pa} \times 10^{-8}$ & $15.1 \pm 0.5$ \\
\hline $\mathrm{DC}, 1 / \mathrm{Pa} \times 10^{-6}$ & $37.8 \pm 12.6$ \\
\hline $\mathrm{AD}, 1 / \mathrm{Pa} \times 10^{-6}$ & $21.5 \pm 7.6$ \\
\hline$E_{\mathrm{p}}, \mathrm{kPa}$ & $52.1 \pm 18.4$ \\
\hline$E_{\text {inc }}, \mathrm{kPa}$ & $172 \pm 133$ \\
\hline$\beta-S I, A U$ & $4.3 \pm 1.4$ \\
\hline $\mathrm{cfPWV}, \mathrm{m} / \mathrm{s}$ & $9.3 \pm 1.9$ \\
\hline
\end{tabular}

BMI, body mass index; PWV, pulse wave velocity; BP, blood pressure; $\mathrm{PP}$, pulse pressure; IMT, intima-media thickness; SD, standard deviation; $\beta$-SI, $\beta$-stiffness index; CC, cross-sectional compliance; AC, arterial compliance; DC, distensibility coefficient; $\mathrm{AD}$, arterial distensibility; $E_{\mathrm{p}}$, Peterson's modulus; $E_{\text {inc }}$, incremental elastic modulus; cfPWV, carotid-femoral PWV.

where $\Delta D$ is the change in arterial diameter, $\Delta P$ is the change in local blood pressure, $D_{\mathrm{d}}$ is the arterial diameter at the end-diastole, IMT is intima-media thickness, and BP is blood pressure where 1 $\mathrm{mm} \mathrm{Hg}=133.322 \mathrm{~Pa}$.

\section{Literature-Based Conversion Equations}

First, we performed the literature search to obtain equations to convert image-based arterial stiffness measures to PWV. Bramwell-Hill PWV equation [10] can be modified to convert DC and cross-sectional compliance into local PWV as follows [1, 20, 21].

$$
\begin{aligned}
& \mathrm{PWV}_{\mathrm{DC}}=\sqrt{\frac{1}{\rho \times \mathrm{DC}}} \\
& \mathrm{PWV}_{\mathrm{CC}}=\sqrt{\frac{\text { Area }}{\rho \times \mathrm{CC}}}
\end{aligned}
$$

where $\rho$ is blood density assumed to be $1,059 \mathrm{~kg} / \mathrm{m}^{3}$.

Moens-Korteweg equation $[22,23]$ can be used to convert $E_{\text {inc }}$ to local PWV [24].

$$
\mathrm{PWV}_{E_{\text {inc }}}=\sqrt{\frac{E_{\text {inc }} \times \mathrm{IMT}}{\rho \times D_{\mathrm{d}}}}
$$

$\beta$-stiffness index can be converted to local PWV using the following equation [26].

$$
\mathrm{PWV}_{\beta-\mathrm{SI}}=\sqrt{\frac{\text { Diastolic BP } \times \beta-\mathrm{SI}}{\rho \times D_{\mathrm{d}}}}
$$

For other measures of arterial stiffness, including $\mathrm{AC}$ and $\mathrm{AD}$, conversion equations were not found in the published literature. Accordingly, we generated mathematical equations converting them to local PWV based on Bramwell-Hill model.

$$
\begin{aligned}
& \mathrm{PWV}_{\mathrm{AC}}=\sqrt{\frac{D_{\mathrm{d}}^{2}}{\rho \times \mathrm{AC} \times\left(2 D_{\mathrm{d}} \times \Delta D\right)}} \\
& \mathrm{PWV}_{\mathrm{AD}}=\sqrt{\frac{D_{\mathrm{d}}}{\rho \times \mathrm{AD} \times\left(2 D_{\mathrm{d}} \times \Delta D\right)}}
\end{aligned}
$$

Regression-based equation can be used to convert $E_{\mathrm{p}}$ to aortic PWV based on regression analysis study [25].

$$
\text { Aortic PWC }(\mathrm{cm} / \mathrm{s})=194.7+5.67 E_{\mathrm{p}}(\mathrm{kPa}) \text {. }
$$

We calculated the values of each local PWV using literaturebased equations. The calculated local PWV was compared against the original arterial stiffness and cfPWV, a reference standard measure of arterial stiffness.

\section{Laboratory-Based Conversion Equations}

For the laboratory-based study, we used cfPWV collected in 49 participants varying in age to generate laboratory-based equations to derive local PWV, that is, cfPWV was used as predictor and ultrasound-derived arterial stiffness measures were utilized as regressors to generate regression equations using the line of the best fit. We assessed strengths of correlations between ultrasoundbased arterial stiffness measures and cfPWV using a variety of equations to derive lines of best fit. The calculated local PWV was compared against the original arterial stiffness and the directly measured cfPWV.

\section{Statistical Analyses}

All local PWV from both literature-based and laboratory-based equations were correlated to cfPWV using univariate correlation analyses and Spearmen's rank correlation analyses. Bland-Altman plot was used to compare between local PWV derived from equations and cfPWV. Local PWV derived from literature-based equations were correlated to local PWV derived from laboratory-based equations using univariate correlation analyses. In all analyses, statistical significance was set a priori at $p<0.05$. SPSS statistics software version 22 (IBM, Chicago, IL) was used for all statistical analyses. Figures and Bland-Altman analyses were constructed with GraphPad Prism 8 (GraphPad Software, San Diego, CA).

\section{Results}

Table 1 shows selected characteristics of the participants. The participants varied widely in age and were apparently healthy.

All local PWV derived from literature-based equations were similar in mean values (Table 2). Local PWV values were strongly correlated with original arterial stiffness measures (the weakest $r$ was 0.81 for $\mathrm{PWV}_{E_{\text {inc }}}$ and the strongest $r$ was 0.99 for $\left.\mathrm{PWV}_{E_{\mathrm{p}}}, p<0.05\right)$. All literaturebased conversion equations were moderately but signifi- 
Table 2. Characteristics of local PWV derived from ultrasound measures of arterial stiffness using literature-based conversion equations

\begin{tabular}{|c|c|c|c|c|}
\hline Local PWV & Equations to derive local PWV & $\begin{array}{l}\text { Mean } \pm S D \\
\mathrm{~m} / \mathrm{s}\end{array}$ & $\begin{array}{l}r \text { between local PWV and } \\
\text { original stiffness }\end{array}$ & $\begin{array}{l}r \text { between local } \\
\text { PWV and cfPWV }\end{array}$ \\
\hline $\mathrm{PWV}_{\mathrm{CC}}$ & $\mathrm{PWV}_{\mathrm{CC}}=\sqrt{\frac{A}{\rho \times \mathrm{CC}}}$ & $5.09 \pm 1.09$ & $-0.85^{*}$ & $0.42 *$ \\
\hline $\mathrm{PWV}_{\mathrm{AC}}$ & $\mathrm{PWV}_{\mathrm{AC}}=\sqrt{\frac{D_{\mathrm{d}}^{2}}{\rho \times \mathrm{AC} \times\left(2 D_{\mathrm{d}}+\Delta D\right)}}$ & $4.79 \pm 0.84$ & $-0.89 *$ & $0.46^{*}$ \\
\hline $\mathrm{PWV}_{\mathrm{DC}}$ & $\mathrm{PWV}_{\mathrm{DC}}=\sqrt{\frac{1}{\rho \times \mathrm{DC}}}$ & $5.18 \pm 0.81$ & $-0.93^{*}$ & $0.41^{*}$ \\
\hline $\mathrm{PWV}_{\mathrm{AD}}$ & $\mathrm{PWV}_{\mathrm{AD}}=\sqrt{\frac{D_{\mathrm{d}}}{\rho \times \mathrm{AD} \times\left(2 D_{\mathrm{d}}+\Delta D\right)}}$ & $4.79 \pm 0.84$ & $-0.92^{*}$ & $0.46^{*}$ \\
\hline $\mathrm{PWV}_{E_{\mathrm{p}}}$ & $\mathrm{PWV}_{E_{\mathrm{p}}}=194.7+5.67 \times E_{\mathrm{p}}$ & $4.9 \pm 1.04$ & $0.99 *$ & $0.43^{*}$ \\
\hline $\mathrm{PWV}_{E_{\text {inc }}}$ & $\mathrm{PWV}_{E_{\mathrm{inc}}}=\sqrt{\frac{E_{\mathrm{inc}} \times \mathrm{IMT}}{\rho \times D_{\mathrm{d}}}}$ & $4.89 \pm 0.83$ & $0.81^{*}$ & $0.41^{*}$ \\
\hline $\mathrm{PWV}_{\beta \text {-SI }}$ & $\mathrm{PWV}_{\beta-\mathrm{SI}}=\sqrt{\frac{\mathrm{DBP} \times \beta-\mathrm{SI}}{2 \rho}}$ & $4.45 \pm 0.77$ & $0.94^{*}$ & $0.50^{*}$ \\
\hline
\end{tabular}

CC, cross-sectional compliance; AC, arterial compliance; DC, distensibility coefficient; AD, arterial distensibility; $E_{\mathrm{p}}$, Peterson's modulus; $E_{\text {inc }}$ incremental elastic modulus; $\beta$-SI, $\beta$-stiffness index; $A$, cross-sectional area at end-diastole; $\rho$, blood density (assumed to be $1,059 \mathrm{~kg} / \mathrm{m}^{3}$ ); $D_{\mathrm{d}}$, arterial diameter at end-diastole; $\Delta D$, the difference between diastolic and systolic diameter; IMT, intima-media thickness; DBP, diastolic blood pressure; PWV, pulse wave velocity; SD, standard deviation. ${ }^{*} p<0.01$.

Table 3. Characteristics of local PWV derived from ultrasound measures of arterial stiffness using laboratory-based conversion equations

\begin{tabular}{|c|c|c|c|c|}
\hline $\mathrm{AC}, \mathrm{mm} / \mathrm{mm} \mathrm{Hg}$ & $\mathrm{PWV}_{\mathrm{AC}}=4.93+0.11(1 / \mathrm{AC})-6.01 \times 10^{-4} \times(1 / \mathrm{AC})^{2}$ & $9.11 \pm 0.59$ & $-0.97^{*}$ & $0.45^{*}$ \\
\hline $\mathrm{DC}, 1 / \mathrm{Pa}$ & $\mathrm{PWV}_{\mathrm{DC}}=5.06+0.0002 \times(1 / \mathrm{DC})-2 \times 10^{-9} \times(1 / \mathrm{DC})^{2}$ & $9.01 \pm 0.64$ & $-0.98^{*}$ & $0.43^{*}$ \\
\hline$E_{\mathrm{p}}, \mathrm{Pa}$ & $\mathrm{PWV}_{E_{\mathrm{p}}}=5.88+65.78 \times 10^{-6} \times E_{\mathrm{p}}$ & $9.30 \pm 1.21$ & $0.99 *$ & $0.49^{*}$ \\
\hline$E_{\text {inc }}, \mathrm{Pa}$ & $\mathrm{PWV}_{E_{\text {inc }}}=8.47+2.127 \times 10^{-6} \times E_{\text {inc }}$ & $9.21 \pm 0.36$ & $0.97^{*}$ & 0.15 \\
\hline$\beta$-SI, AU & $\mathrm{PWV}_{\beta-\mathrm{SI}}=7.45+0.38 \times \beta-\mathrm{SI}$ & $9.04 \pm 0.57$ & $0.88^{*}$ & $0.39 *$ \\
\hline
\end{tabular}

CC, cross-sectional compliance; AC, arterial compliance; DC, distensibility coefficient; AD, arterial distensibility, $E_{\mathrm{p}}$, Peterson's modulus; $E_{\text {inc }}$ incremental elastic modulus; $\beta$-SI, $\beta$-stiffness index; PWV, pulse wave velocity; cfPWV, carotid-femoral PWV; SD, standard deviation. ${ }^{*} p<0.01$. 
cantly correlated with directly measured cfPWV ( $r$ ranging from 0.41 for $P W V_{D C}$ to 0.50 for $\left.P W V_{\beta-S I}\right)$. In order to derive better fits, many different plots (e.g., curvilinear plots) were fit into the model. However, these models did not produce significantly better fits, these plots were dropped.

As can be seen in Table 3, mean PWV values derived from the regression equations were similar ranging from 9.01 to $9.30 \mathrm{~m} / \mathrm{s}$. The local PWV using regression-based equations were strongly correlated with original local arterial stiffness ( $r$ ranged from 0.88 to 0.99 , all $p<0.01$ ) and local PWV derived from literature-based equations $(r$ ranged from 0.77 to 0.99 ) (Table 4). The local PWV derived from ultrasound-based arterial stiffness was modestly but significantly associated with cfPWV. The only exception was incremental elastic modulus as it was not correlated with cfPWV $(r=0.15, p>0.05)$.

Figures 1 and 2 show Bland-Altman plots comparing derived local PWV and cfPWV. The mean differences and standard deviations of error for the literature-based $\mathrm{PWV}$ ranged from $4.2 \pm 1.4 \mathrm{~m} / \mathrm{s}$ for $\mathrm{PWV}_{E_{\text {inc }}}$ to $4.6 \pm 1.3$ $\mathrm{m} / \mathrm{s}$ for $\mathrm{PWV}_{\beta \text {-SI }}$ (Fig. 1). On the other hand, the mean differences and standard deviation of error for PWV derived from the regression equations ranged from $0.0 \pm 1.4$ $\mathrm{m} / \mathrm{s}$ for $\mathrm{PWV}_{\mathrm{CC}}$ to $-0.2 \pm 1.4 \mathrm{~m} / \mathrm{s}$ for $\mathrm{PWV}_{E_{\mathrm{p}}}$. Figures 3 and 4 show intercorrelations between local PWV derived from literature-based equations, regression-based equations, and cfPWV.

\section{Discussion}

The purpose of the present study was to identify, create, and evaluate conversion equations to transform common image-based measures of arterial stiffness to local PWV. We reasoned that the availability of such equations will facilitate meta-analyses and systemic reviews of arterial stiffness since a variety of arterial stiffness indices have been used in different research studies. The main conclusion is that all the image- or ultrasound-based local arterial stiffness evaluated in the present study can be converted to local PWV using either the literature-based or the laboratory-generated regression equations. These converted local PWV were significantly correlated to cfPWV, a reference standard measure of arterial stiffness.

Even though cfPWV has been considered the reference standard measure of arterial stiffness, a variety of other methods have been used to measure arterial stiffness [8]. Concerns have been raised that a lack of standardization of methodologies could underpower the systematic data analysis as each methodology evaluates different aspects
Table 4. The associations between local PWV derived from the literature-based equations and the regression-based equations and cfPWV

\begin{tabular}{llll}
\hline $\begin{array}{l}\text { Arterial } \\
\text { stiffness }\end{array}$ & $\begin{array}{l}\text { Spearman's } r \\
\text { between the } \\
\text { literature-based } \\
\text { PWV and cfPWV }\end{array}$ & $\begin{array}{l}\text { Spearman's } r \\
\text { between the } \\
\text { regression-based } \\
\text { PWV and cfPWV }\end{array}$ & $\begin{array}{l}\text { Pearson's } r \text { between } \\
\text { the literature-based } \\
\text { and regression-based } \\
\text { PWV }\end{array}$ \\
\hline CC & $0.53^{*}$ & $0.43^{*}$ & $0.84^{*}$ \\
AC & $0.53^{*}$ & $0.48^{*}$ & $0.89^{*}$ \\
$\mathrm{DC}$ & $0.45^{*}$ & $0.45^{*}$ & $0.94^{*}$ \\
$\mathrm{AD}$ & $0.53^{*}$ & $0.52^{*}$ & $0.98^{*}$ \\
$E_{\mathrm{p}}$ & $0.51^{*}$ & $0.51^{*}$ & $0.99^{*}$ \\
$E_{\text {inc }}$ & $0.51^{*}$ & 0.13 & $0.77^{*}$ \\
$\beta-$ SI & $0.58^{*}$ & $0.42^{*}$ & $0.94^{*}$ \\
\hline
\end{tabular}

$* p<0.01$. CC, cross-sectional compliance; AC, arterial compliance; $\mathrm{DC}$, distensibility coefficient; $\mathrm{AD}$, arterial distensibility, $E_{\mathrm{p}}, \mathrm{Pe}$ terson's modulus; $E_{\text {inc }}$, incremental elastic modulus; $\beta$-SI, $\beta$-stiffness index; PWV, pulse wave velocity; cfPWV, carotid-femoral PWV.

in the arterial tree and is expressed using different unit and methodology [13-17]. For instance, in a meta-analysis aimed at evaluating the effect of resistance exercise training on arterial stiffness, the investigators limited the included studies to these reporting PWV [13-15]. In addition, random effect models were used because of the heterogenous outcome between the different studies [17]. In another meta-analysis [16], this particular issue was mitigated by standardizing mean difference among different arterial stiffness measures between pre- and postexercise intervention. A variety of regression equations presented herein can be used to convert image-based arterial stiffness measures into local PWV using the same unit.

As an initial step, the literature search was conducted to identify conversion equations that can be used to transform imaging-based arterial stiffness measures to local PWV. Using a variety of previously published formulas, including the Bramwell-Hill equation [10] and the Moens-Korteweg equation $[22,23]$ we were able to convert all the commonly used image-based measures of arterial stiffness to local PWV. One problem of the literature-based equations, however, is that they require variables that are not typically reported or presented in previously published articles. Depending upon regression equations, these variables include arterial wall distension, arterial diameter at end-diastole, local diastolic blood pressure at the site of arterial stiffness measurement (e.g., carotid artery), and arterial wall intima-media thickness. In the similar context, equations that include age might produce better regression equations [25] as age 

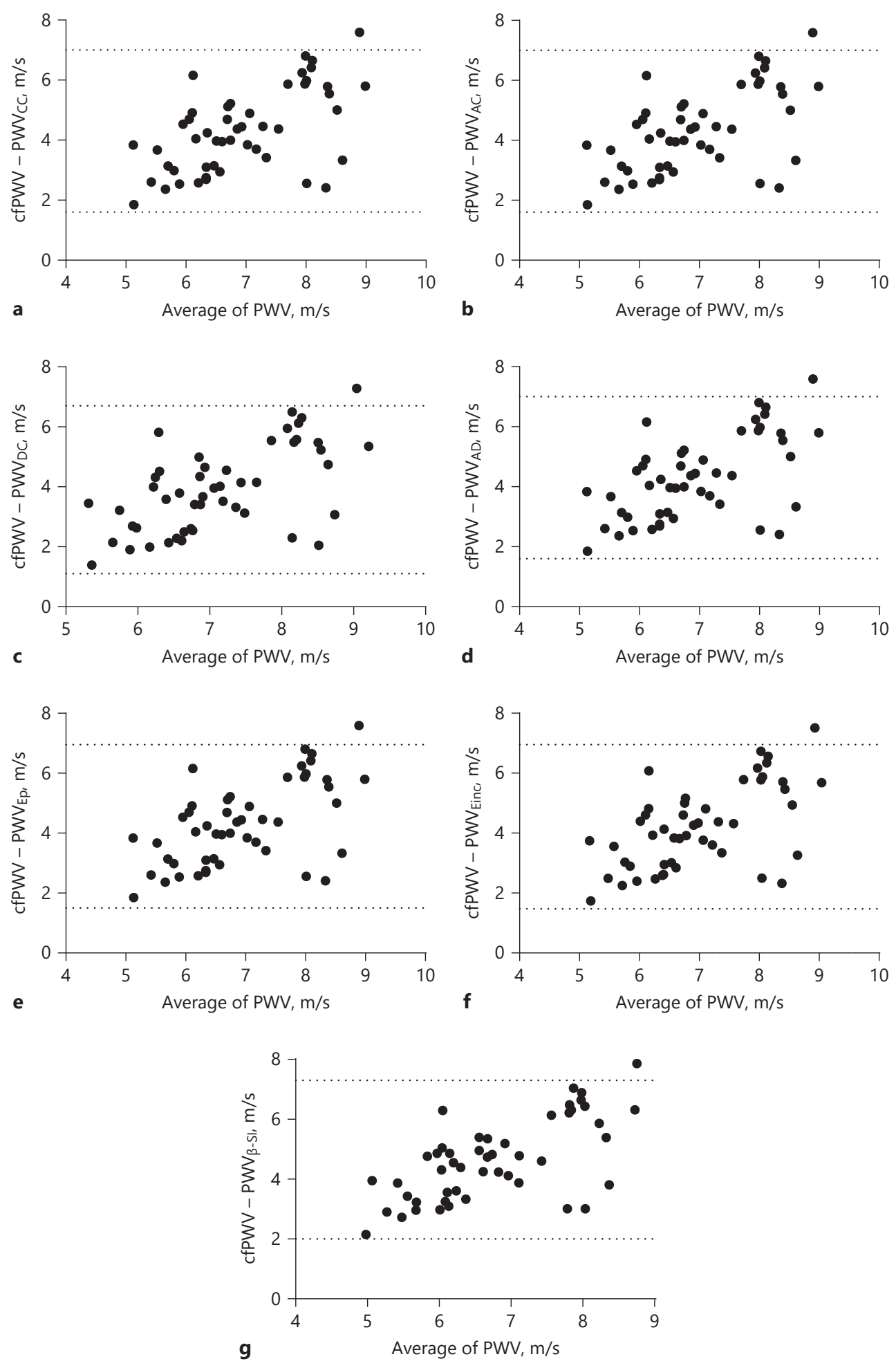

Fig. 1. Bland-Altman plot showing associations between the literature-based local PWV and cfPWV. Mean differences \pm 2 SDs are displayed. CC, cross-sectional compliance (a), AC, arterial compliance (b), DC, distensibility coefficient $(\mathbf{c}), \mathrm{AD}$, arterial distensibility $(\mathbf{d}), E_{\mathrm{p}}$, Peterson's modulus $(\mathbf{e}), E_{\text {inc }}$, incremental elastic modulus $(\mathbf{f})$, $\beta$-SI, $\beta$-stiffness index (g). PWV, pulse wave velocity; cfPWV, carotid-femoral PWV; SD, standard deviation.

Standardization of Arterial Stiffness Measures
Pulse 2021:9.72-82

DOI: $10.1159 / 000517872$ 

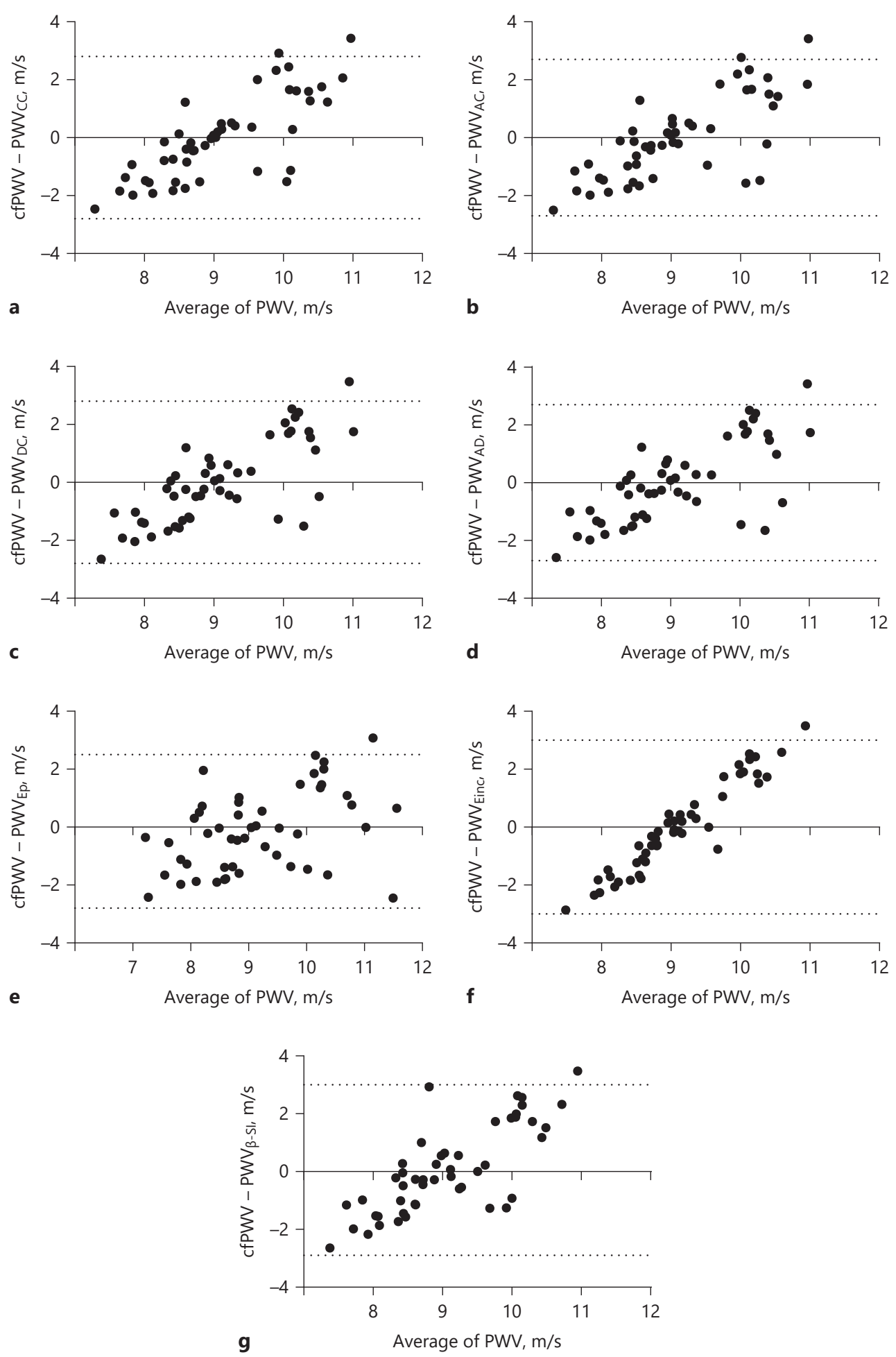

Fig. 2. Bland-Altman plot showing associations between the laboratory-based PWV and cfPWV. Mean differences \pm 2 SDs are displayed. CC, cross-sectional compliance (a), AC, arterial compliance (b), DC, distensibility coefficient $(\mathbf{c}), \mathrm{AD}$, arterial distensibility $(\mathbf{d}), E_{\mathrm{p}}$, Peterson's modulus $(\mathbf{e}), E_{\text {inc }}$, incremental elastic modulus (f), $\beta$-SI, $\beta$-stiffness index (g). PWV, pulse wave velocity; cfPWV, carotid-femoral PWV; SD, standard deviation. 

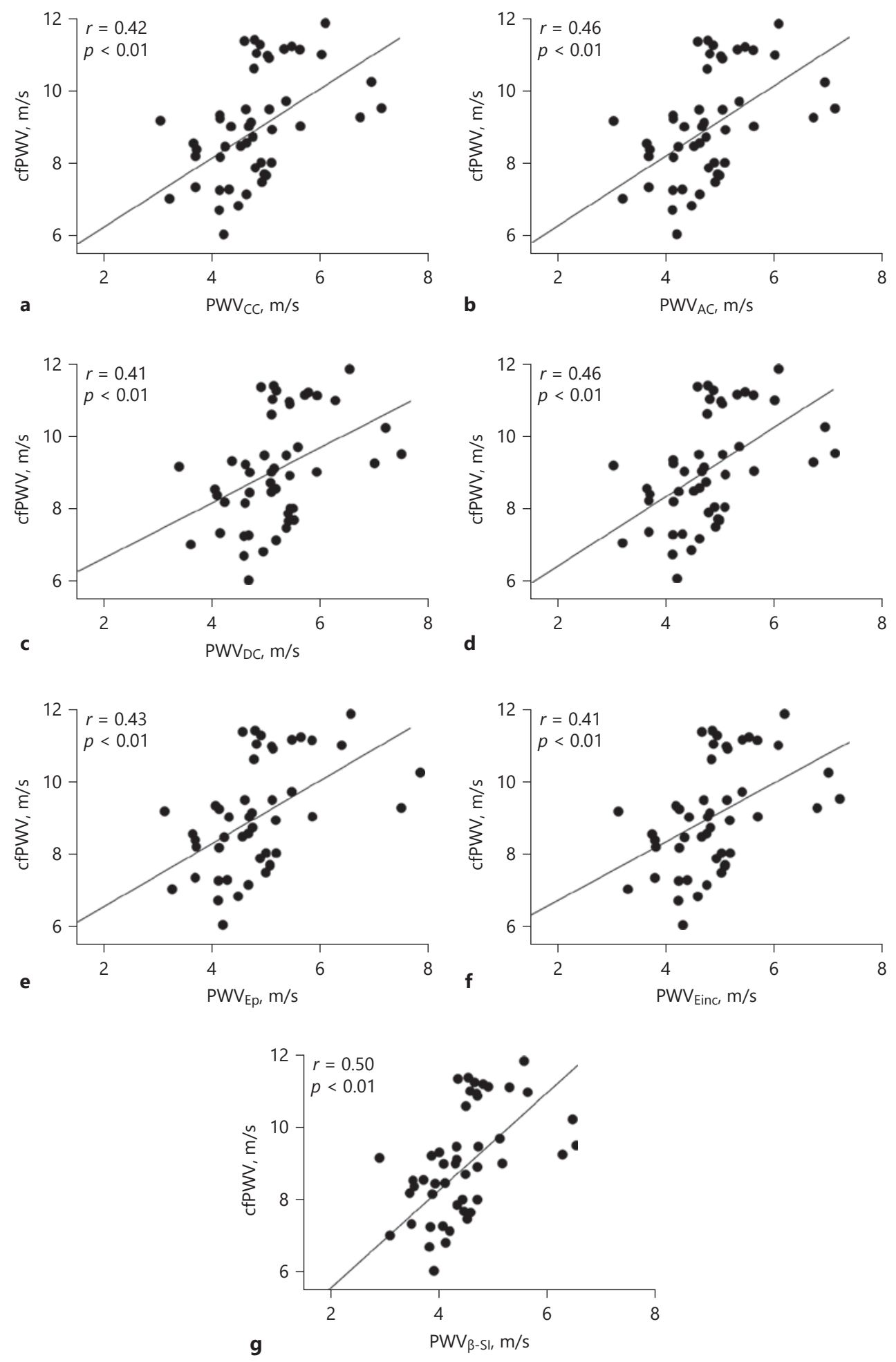

Fig. 3. Correlation and regression plots showing the associations between laboratory-based local PWV and cfPWV. CC, cross-sectional compliance (a), AC, arterial compliance (b), DC, distensibility coefficient (c), AD, arterial distensibility $(\mathbf{d}), E_{\mathrm{p}}$, Peterson's modulus $(\mathbf{e}), E_{\text {inc }}$, incremental elastic modulus (f), $\beta$-SI, $\beta$-stiffness index $(\mathbf{g})$. PWV, pulse wave velocity; cfPWV, carotid-femoral PWV; SD, standard deviation. 

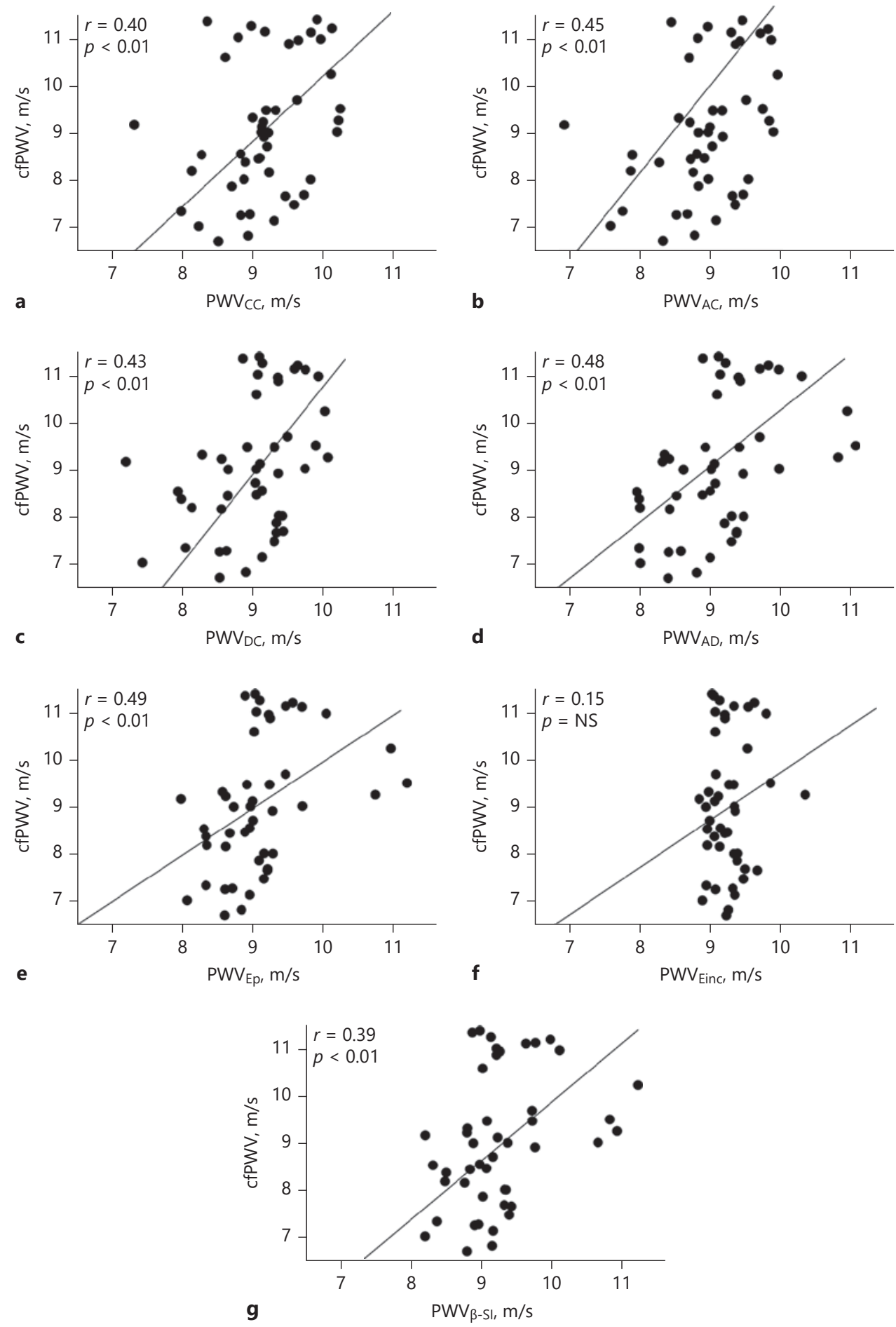

Fig. 4. Correlation and regression plots showing the associations between regression-based local $\mathrm{PWV}$ and cfPWV. CC, cross-sectional compliance (a), AC, arterial compliance (b), DC, distensibility coefficient (c), AD, arterial distensibility $(\mathbf{d}), E_{\mathrm{p}}$, Peterson's modulus $(\mathbf{e}), E_{\text {inc }}$, incremental elastic modulus (f), $\beta$-SI, $\beta$-stiffness index (g). PWV, pulse wave velocity; cfPWV, carotid-femoral PWV; SD, standard deviation. 
is strongly associated with arterial stiffness. However, ages of individual groups or subgroups are often not reported even though the average age of the whole sample is always reported. Accordingly, we also generated laboratory-based conversation equations that only require arterial stiffness values to convert them to local PWV.

Local PWV derived from the literature-based equations $(\sim 4-5 \mathrm{~m} / \mathrm{s})$ were consistently lower than directly measured cfPWV $(\sim 9 \mathrm{~m} / \mathrm{s})$. In a previous study comparing local PWV obtained at the carotid artery and cfPWV, local PWV was much lower than regional PWV [29]. This is not surprising as PWV is known to vary widely on the arterial tree having a lower PWV value at a more proximal location (e.g., common carotid artery). In a study, using simultaneous micromanometric pressure measurements, PWV changes from $4.4 \mathrm{~m} / \mathrm{s}$ at the aortic root, $5.7 \mathrm{~m} / \mathrm{s}$ at the abdominal aorta, to $8.8 \mathrm{~m} / \mathrm{s}$ at the femoral artery [30]. On the other hand, local PWV derived from the laboratory-based equations were regressed to cfPWV and accordingly have values of PWV that are closer to cfPWV with lower mean differences between local PWV and cfPWV as tested by the Bland-Altman plots.

Local arterial stiffness was significantly associated with segmental PWV as shown in previous studies [20,31]. However, local PWV calculated from literature-based equations were much lower than cfPWV. There are a number of explanations for this. Locally measured AC reflects pulsatile function at a highly elastic artery while cfPWV is a segmental measure of arterial stiffness that derived from the velocity of pulse wave traveling along the arterial tree [8]. Arterial cushioning functions change along the arterial tree [30]. For example, differences in chemical composition may alter mechanical properties throughout the arterial tree as the proportion of elastin content decreases from the aorta to more peripheral arteries [32]. In this context, cfPWV reflects mostly the stiffness of central elastic arteries but also include arterial stiffness of peripheral muscular arteries (i.e., femoral artery) [30].

A previous study using cardiovascular magnetic reasoning imaging measured and compared DC and simultaneously measured aortic PWV [20]. DC was significantly associated with cfPWV with Pearson's correlation of $r=0.69$. In the present study, derived local PWV reflects local arterial stiffness of the carotid artery whereas cfPWV is a regional arterial stiffness ranging from the carotid to femoral arteries. In spite of these differences, we found significant and modest associations between local PWV and cfPWV. Taken together, these results suggest that the derived local PWV can provide quantitatively similar information to directly measured PWV.

Standardization of Arterial Stiffness

Measures
There are a number of limitations that we should discuss. First, in order to minimize confounding influences of disease states, the present study was performed on apparently healthy adults varying widely in age. Thus, the generalizability of our findings may be limited even though this was a "cleaner" approach to minimize confounding influences of various diseases. Second, the measurement modalities of arterial stiffness are extremely diverse. We chose to focus on 2 of the most commonly used measures of arterial stiffness: image-based local arterial stiffness and PWV. Other measures of arterial stiffness including Windkessel model (e.g., C1 and C2 measured with the HDI device) and oscillometric-based assessment [8] were not included in the present study. Third, incremental elastic modulus was not significantly correlated to cfPWV. However, this issue could be resolved by converting incremental elastic modulus to Peterson's modulus by multiplying incremental elastic modulus by $2 \mathrm{MT} / \mathrm{Dd}$ then using the result in Peterson's elastic modulus converting equations if wall thickness and diastolic diameter are known. Fourth, because the association between PWV and local measures of arterial stiffness can be curvilinear [6], the plotting based on linear regressions may not be appropriate in some instances. However, in these cases, curvilinear plots were not significantly better than linear regression lines as linear regression lines were the lines of best fit from the statistical standpoint. Last, the number of participants studied is relatively small compared with large epidemiological studies $[27,28]$ that these equations are intended to be used.

In summary, all the ultrasound-based local arterial stiffness evaluated in the present study can be converted to local PWV using either the literature-based or the laboratory-generated regression equations. These converted local PWV were significantly correlated to cfPWV. We reasoned that these equations can be used in meta-analyses and systematic reviews to express arterial stiffness in the same unit and in the similar scale to compare group differences in various populations and effectiveness of various interventions.

\section{Acknowledgment}

We acknowledge the contribution of Brandon Fico who helped and reviewed this manuscript.

\section{Statement of Ethics}

The Institutional Review Board at University of Texas at Austin approved this study (IRB\#2014-02-0015). Written informed consent was obtained from all participants. 


\section{Conflict of Interest}

The authors declared no conflict of interest.

\section{Funding Sources}

The authors did not receive any funding.

\section{Author Contributions}

Jisok Lim recruited the participants and run all arterial stiffness measures, Taha Alhalimi runs the literature research and the statistical analyses, Drew Gourley revised the manuscript and the statistical analyses, and Hirofumi Tanaka devised, revised, and supervised the whole project. All the authors provided critical analyses on the manuscript and approved the final version.

\section{Data Availability Statement}

Datasets generated during the current study will be available on reasonable request.

\section{References}

1 Townsend RR, Wilkinson IB, Schiffrin EL, Avolio AP, Chirinos JA, Cockcroft JR, et al. Recommendations for improving and standardizing vascular research on arterial stiffness: a scientific statement from the American Heart Association. J Hypertens. 2015;66: 698-722.

2 Mora S, Cook N, Buring JE, Ridker PM, Lee IM. Physical activity and reduced risk of cardiovascular events: potential mediating mechanisms. Circ J. 2007;116:2110-8.

3 Paffenbarger RS, Hyde RT, Wing AL, Hsieh CC. Physical activity, all-cause mortality, and longevity of college alumni. N Engl J Med. 1986;314(10):605-13.

4 Hayashi K, Yamottoto T, Takahara A, Shirai K. Clinical assessment of arterial stiffness with cardio-ankle vascular index: theory and applications. J Hypertens. 2015;39:1742-57.

5 Rabben SI, Stergiopulos N, Hellevik LR, Smiseth OA, Slørdahl S, Urheim S, et al. An ultrasound-based method for determining pulse wave velocity in superficial arteries. J Biomech. 2004;37(10):1615-22.

6 Lim J, Pearman M, Park W, Alkatan M, Tana$\mathrm{ka} \mathrm{H}$. Interrelationships among various measures of central artery stiffness. Am J Hypertens. 2016;29(9):1024-8

7 Reference Values for Arterial Stiffness' Collaboration. Determinants of pulse wave velocity in healthy people and in the presence of cardiovascular risk factors: 'establishing normal and reference values. Eur Heart J. 2010; 31:2338-50.

8 Tanaka H. Various indices of arterial stiffness: are they closely related or distinctly different? Pulse. 2018;5:1-6.

9 Van Bortel LM, Duprez D, Starmans-Kool MJ, Safar ME, Giannattasio C, Cockcroft J, et al. Clinical applications of arterial stiffness, task force III: recommendations for user procedures. Am J Hypertens. 2002;15(5):445-52.

10 Bramwell JC, Hill AV. The velocity of pulse wave in man. Proc R Soc Lond B. 1922; 93(652):298-306.

11 Tanaka H, DeSouza CA, Seals DR. Arterial stiffness and hormone replacement use in healthy postmenopausal women. J Gerontol A Biol Sci Med Sci. 1998;53(5):M344-6.
12 Rhee M-Y, Lee H-Y, Park JB, Park JB. Measurements of arterial stiffness: methodological aspects. Korean Circ J. 2008;38(7):343-50.

13 Miyachi M. Effects of resistance training on arterial stiffness: a meta-analysis. Br J Sports Med. 2013;47(6):393-6.

14 Rodríguez AJ, Scott D, Srikanth V, Ebeling P. Effect of vitamin D supplementation on measures of arterial stiffness: a systematic review and meta-analysis of randomized controlled trials. Clin Endocrinol. 2016;84(5):645-57. 5

15 Upala S, Sanguankeo A, Congrete S, Jaruvongvanich V. Effect of cholecalciferol supplementation on arterial stiffness: a systematic review and meta-analysis. Scand Cardiovasc J. 2016;50(4):230-5.

16 Montero D, Roche E, Martinez-Rodriguez A. The impact of aerobic exercise training on arterial stiffness in pre- and hypertensive subjects: a systematic review and meta-analysis. Int J Cardiol. 2014;173(3):361-8.

17 Montero D, Roberts CK, Vinet A. Effect of aerobic exercise training on arterial stiffness in obese populations: a systematic review and meta-analysis. Sports Med. 2014;44:833-43.

18 Cortez-Cooper MY, Supak JA, Tanaka H. A new device for automatic measurements of arterial stiffness and ankle-brachial index. Am J Cardiol. 2003;91(12):1519-22.

19 DeVan AE, Anton MM, Cook JN, Neidre DB, Cortez-Cooper MY, Tanaka H. Acute effects of resistance exercise on arterial compliance. J Appl Physiol (1985). 2005;98(6):2287-91.

20 Dogui A, Kachenoura N, Frouin F, Lefort M, De Cesare A, Mousseaux E, et al. Consistency of aortic distensibility and pulse wave velocity estimates with respect to the Bramwell-Hill theoretical model: a cardiovascular magnetic resonance study. J Cardiovasc Magn Reson. 2011;13:11.

21 Engelen L, Bossuyt J, Ferreira I, van Bortel LM, Reesink KD, Segers P, et al. Reference values for local arterial stiffness. Part A: carotid artery. J Hypertens. 2015;33(10): 198196.

22 Moens AI. Die pulscurve. Leiden: E J Brill; 1878. p. 87-95.
23 Korteweg DJ. Ueber die Fortpflanzungsgeschwindigkeit des Schalles in elastischen Röhren. Ann Phys Chem. 1878;241(12):52542.

24 Callaghan FJ, Geddes LA, Babbs CF, Bourland JD. Relationship between pulse-wave velocity and arterial elasticity. Med Biol Eng Comput. 1986;24(3):248-54.

25 Nagai Y, Fleg JL, Kemper MK, Rywik TM, Earley CJ, Metter EJ. Carotid arterial stiffness as a surrogate for aortic stiffness: relationship between carotid artery pressure-strain elastic modulus and aortic pulse wave velocity. Ultrasound Med Biol. 1999;25:181-8.

26 Zieff GH, Heffernan K, Stone K, Fryer S, Credeur D, Hanson ED, et al. The pressure-dependency of local measures of arterial stiffness. Hypertension. 2019;37(5):956-63.

27 Arnett DK, Chambless LE, Kim H, Evans GW, Riley W. Variability in ultrasonic measurements of arterial stiffness in the atherosclerosis risk in communities study. Ultrasound Med Biol. 1999;25(2):175-80.

28 Tanaka H, Heiss G, McCabe EL, Meyer ML, Shah AM, Mangion JR, et al. Hemodynamic correlates of blood pressure in older adults: the atherosclerosis risk in communities (ARIC) study. J Clin Hypertens. 2016;18(12): 1222-7.

29 Simova I, Katova T, Santoro C, Galderisi M, Galderisi M. Comparison between regional and local pulse-wave velocity data. Echocardiography. 2016;33(1):77-81.

30 Latham RD, Westerhof N, Sipkema P, Rubal BJ, Reuderink P, Murgo JP. Regional wave travel and reflections along the human aorta: a study with \six simultaneous micromanometric pressures. Circulation. 1985;72:125769.

31 Marque V, van Essen H, Struijker-Boudier HA, Atkinson J, Lartaud-Idjouadiene I. Determination of aortic elastic modulus by pulse wave velocity and wall tracking in a rat model of aortic stiffness. J Vasc Res. 2001 Nov-Dec; 38(6):546-50.

32 Harkness ML, Harkness RD, Mcdonald DA. The collagen and elastin content of the arterial wall in the dog. Proc R Soc Lond B Biol Sci. 1957;146(925):541-51. 